Chapter 16

\title{
Fabrication of PGA/PLA Scaffold with the Shape of Human Nose
}

\author{
Qiong Li, Lu Zhang, Guangdong Zhou, Wei Liu and \\ Yilin Cao
}

Additional information is available at the end of the chapter

http://dx.doi.org/10.5772/55540

\section{Introduction}

Reconstructive surgery for the repair of nose deformities is challenging [1]. Nasal surgery involves autologous rib or septum cartilage grafts [2] and prosthetic devices [3] for reconstruction and reinforcement of the nasal skeleton. These conventional procedures are associated with donor site morbidity, limited tissue availability, and prosthesis related infection and extrusion [4]. Although tissue engineering is a promising method for repair and reconstruction of cartilage defects [5-7], engineering cartilage with a delicate three dimensional (3D) structure, such as human nose, remains a great challenge in this field. Since in 1997 Cao et al. engineered the cartilage with a shape of human auricle in a nude mouse model [8], many researchers have tried to explore further developments of this tissue engineering system, but few of them have succeeded in in vitro regeneration of a cartilage construct with a complete and anatomically refined structure [9].

One major reason leading to the failure of in vitro engineering a cartilage construct with sufficient control over shape is the lack of appropriate scaffolds. The optimal scaffold used for engineering a cartilage construct with accurate designed shapes should possess at least three characteristics: good biocompatibility for cell seeding, ease of being processed into a specific shape, and sufficient mechanical strength for retaining the pre-designed shape. Polyglycolic acid (PGA) has proven to be one of the most successful scaffolds for cartilage regeneration [1012]. Cartilage engineered with the PGA scaffold has structure and composition similar to the native tissue, as demonstrated by histological analysis and cartilage specific matrices [13-15]. However, the most widely used form of PGA material in cartilage engineering is unwoven fiber mesh, which is difficult to be initially prepared into a complicated 3D structure and would most likely fail to maintain its original architecture during subsequent in vitro chondrogenesis due to insufficient mechanical support [14, 16, 17]. 
To overcome these problems, two crucial issues should be addressed. First, the PGA-based scaffold should be prefabricated into the exact shape of human nose. Second, the mechanical strength of the above-mentioned scaffold should be further enhanced so that it can retain the pre-designed shape.

In order to meet these requirements, in the current study, a computer aided design and manufacturing (CAD/CAM) technique was employed to fabricate a set of negative molds, which was then used to press the PGA fibers into the pre-designed nose structure. Furthermore, the mechanical strength of the scaffold was enhanced by coating the PGA fibers with an optimized amount of PLA.

\section{Materials and methods}

\subsection{Preparation of scaffolds with different PLA contents}

$10 \mathrm{mg}$ of unwoven PGA fibers (provided by Dong Hua University, Shanghai, China) were compressed into a cylinder shape of $5 \mathrm{~mm}$ in diameter and $2 \mathrm{~mm}$ in thickness. A solution of 0.3 $\%$ PLA (Sigma, St. Louis, MO, USA) in dichloromethane was evenly dropped onto the PGA scaffold, dried in a $65^{\circ} \mathrm{C}$ oven, and weighed. The PLA mass ratio was calculated according to the formula: PLA $\%=$ (final mass-original mass)/final mass $\times 100 \%$. The above procedures were repeated until the predetermined PLA mass ratios of $0 \%, 10 \%, 20 \%, 30 \%, 40 \%$ and $50 \%$ were achieved. The scaffolds were examined by SEM (Philips XL-30, Amsterdam, Netherlands) [18].

\subsection{Biocompatibility evaluation of the scaffolds}

Cell seeding: Chondrocytes were isolated from the articular cartilage of newborn swine (2-3weeks old) as previously described [19]. The harvested chondrocytes were adjusted to a final concentration of $50 \times 10^{6}$ cells $/ \mathrm{mL}$, and a 100uL cell suspension was pippeted onto each scaffold. The cell-scaffold constructs were then incubated for $4 \mathrm{~h}$ at $37^{\circ} \mathrm{C}$ with $95 \%$ humidity and $5 \% \mathrm{CO}_{2}$ to allow for complete adhesion of the cells to the scaffolds.

Cell adhesion: After 4 hours of incubation, the cell-scaffold constructs were gently transferred into a new 6-well plate. The remaining cells were collected and counted. The cell seeding efficiencies of the scaffolds with different PLA contents were calculated based on the formula: (total cell number- remaining cell number)/ total cell number $\times 100 \%$ [14].

\subsection{Mold fabrication by CAD/CAM}

A patient's normal nose was scanned by CT to obtain the geometric data (Figure 3). These data were further processed by a CAD system to generate both positive and negative of the normal nose, and the resultant data were input into a CAM system (Spectrum 510, Z Corporation) for the fabrication of the resin models by $3 \mathrm{D}$ printing. The negative mold was composed of two parts: the anterior part and the posterior part. (Figure $4 \mathrm{~A}$ ) 


\subsection{Fabrication of nose shaped scaffold}

Two hundred milligrams of unwoven PGA fibers were pressed using the negative mold for over 12 hours. A solution of 0.3 \% PLA (Sigma, St. Louis, MO, USA) in dichloromethane was evenly dropped onto the PGA scaffold, dried in a $65^{\circ} \mathrm{C}$ oven, weighed, and pressed again with the negative mold. This procedure was repeated until the final PLA mass ratio of $20 \%$ was reached. The edge of the scaffold was carefully trimmed according to the shape of the positive mold.

\subsection{Statistical analysis}

The differences of cell seeding efficiencies $(n=6)$ among the six PLA content groups were analyzed using the Student's t-test. A $p$-value less than 0.05 was considered statistically significant.

\section{Results}

\subsection{SEM observation of the scaffolds with different PLA contents}

PLA/PGA scaffold compositions were visualized under SEM. (Figure 1) The pure PGA scaffold (0\% PLA added) appeared as a smooth fiber mesh. In PGA scaffolds supplemented with $10 \%$ PLA, the PLA coating can be seen connecting some fibers, particularly at nodes where PGA fibers cross. In the $20 \%$ PLA embedded scaffold, most mesh nodes visualized were covered with PLA.30\% PLAscaffold had notonly mostmeshnodesembedded in PLA, butalso the PLAcoating was seen covering small portions of the mesh itself, minimally obstructing the porosity of the fiber network. In the $40 \%$ PLA embedded scaffold, most of the mesh porosity is obscured by a PLA. In the $50 \%$ PLA scaffold, the mesh is almost completely obscured by a PLA sheet.

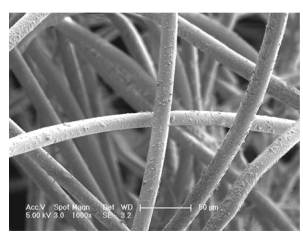

0

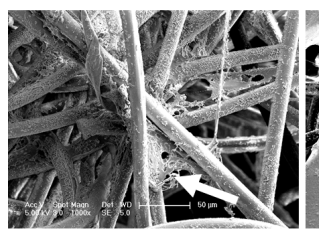

30

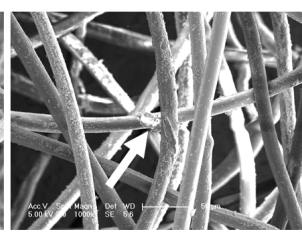

10

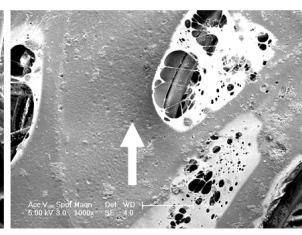

40

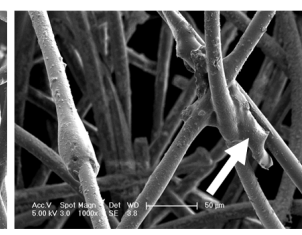

20

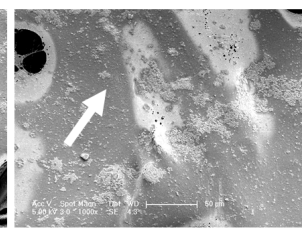

50

Figure 1. SEM examination. Scaffolds with different PLA contents $(0 \%, 10 \%, 20 \%, 30 \%, 40 \%$ and $50 \%)$ show different pore structures. The white arrows indicate the coated PLA. 


\subsection{Evaluation of the biocompatibility of the scaffolds with different PLA contents}

Cell seeding efficiencies were performed to evaluate the influence of PLA contents on cell compatibility of the scaffolds. The results showed that the increase in PLA content could lead to the reduction in the ability of the scaffolds to absorb the cell suspensions (Figure 2A). Quantitative analysis (Figure 2B) demonstrated that all the groups with PLA presented significantly lower cell seeding efficiencies compared to the group without PLA ( $\mathrm{p}<0.05)$. If the acceptable cell adhesion rate is defined over $80 \%$, these results indicate that $20 \%$ but not $30 \%$ is an acceptable PLA amount for preparing the scaffolds in terms of cell seeding efficiency.
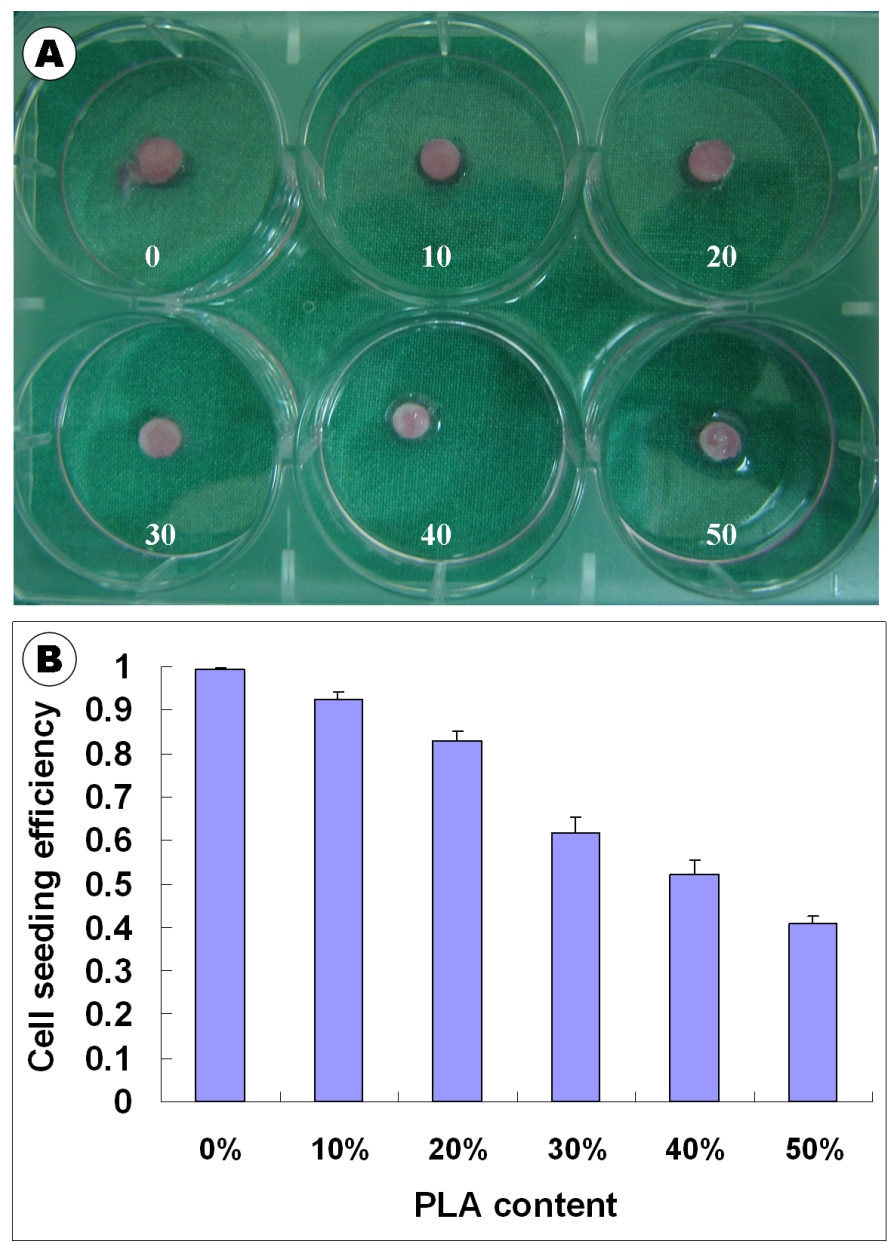

Figure 2. The influences of PLA contents on cell seeding efficiency. (A): Scaffolds with different PLA contents absorb different volumes of the cell suspension. (B): Cell seeding efficiencies decrease with increasing PLA contents in the scaffolds with significant decreases $(p<0.05)$. 


\subsection{Mold preparation and fabrication of the nose-shaped scaffold}

Because good biocompatibility could be achieved in the scaffold with $20 \%$ PLA, this formulation was further used for the fabrication of the human nose shaped scaffold. In order to prepare the scaffold into a shape of normal nose, a set of negative molds was produced according to image of the normal nose (Figure 3). The resulting nose-shaped scaffold (Figure $4 \mathrm{C}$ ) achieved a precise shape compared to its positive mold (Figure 4B). These results indicate that the mold produced by CAD/CAM technology is allowed to accurately fabricate a scaffold into a nose shape.

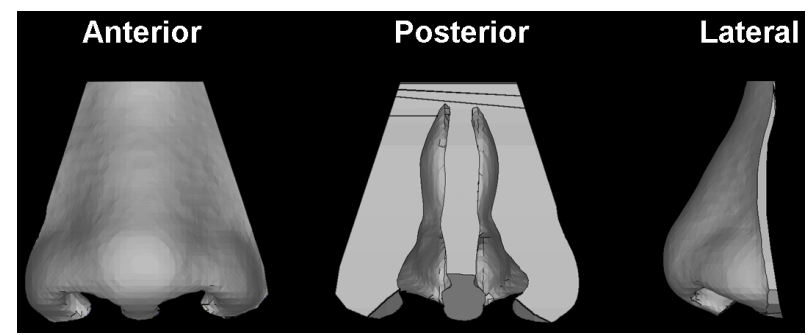

Figure 3. image of a patient's normal nose.
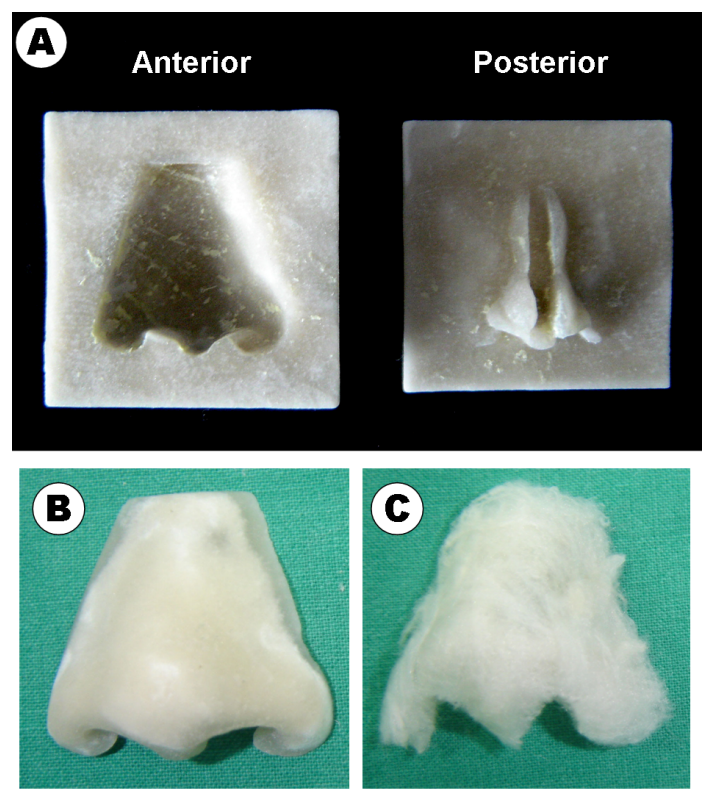

Figure 4. Mold preparation and the fabrication of the nose-shaped scaffolds. (A): The resin negative mold: anterior part and posterior part; (B): The resin positive mold; (C): the nose-shaped PLA/PGA scaffold. 


\section{Discussions}

Despite the rapid progress in cartilage engineering, in vitro engineering of cartilage with a fine controlled 3D structure, such as human nose, remains a great challenge due to the lack of appropriate scaffolds. PGA has proven to be one of the most successful scaffolds for cartilage regeneration. However, for in vitro engineering of a cartilage with a precise shape, PGA unwoven fibers (the most widely used physical form) still have some drawbacks, such as the difficulties in controlling an accurate shape.

To achieve this, a negative mold corresponding to the desired shape is required. CAD/CAM, as a novel technique, has been widely used for the fabrication of anatomically accurate 3D models [20-23]. Particularly, this method can accurately perform complicated manipulations of the original 3D data, including Boolean operations, mirror imaging, and scaling [24- 26]. $\mathrm{CAD} / \mathrm{CAM}$ technique was therefore used in the current study for the production of the negative mold for a human nose. Using this mold, PGA fibers were able to be accurately prepared into the nose-shaped scaffold.

The mechanical strength of PGA scaffold alone is not sufficient for the shape maintenance, and thus PLA coating was used to strengthen its mechanical properties as reported [10, 11, 27]. However, a high amount of PLA in the scaffold would negatively affect cartilage formation because of poor cell compatibility [14]. Therefore, an appropriate PLA content in the scaffold is important for both shape maintenance and biocompatibility. In the current study, we evaluated the effects of six PLA contents on the scaffolds' biocompatibility. According to the current results, although the mechanical strength of the scaffolds increased with increasing PLA content, $20 \%$ is an acceptable PLA amount for preparing the scaffolds in terms of cell seeding efficiency.

Finally, aided by CAD/CAM technique, the PGA fibers were prepared into the accurate shape of a human nose. Furthermore, by coating with PLA, the scaffold could obtain sufficient mechanical strength to retain the original shape. These results may provide useful information for future nose reconstructions by in vitro engineered cartilage as well as for the engineering of other tissues with complicated 3D structures.

In summary, this study established a method to precisely engineer a PGA/PLA scaffold with the shape of human nose. In future studies, we will also investigate the fate of these scaffolds after cell seeding, especially subcutaneous implantation in an immunocompetent animal model.

\section{Acknowledgements}

This research was supported by National Natural Science Foundation of China (81101438 and 81201476). 


\section{Author details}

Qiong Li, Lu Zhang*, Guangdong Zhou, Wei Liu and Yilin Cao

*Address all correspondence to: luzhangmd@gmail.com

Department of Plastic and Reconstructive Surgery, Shanghai th People's Hospital, Shanghai Jiao Tong University School of Medicine, Shanghai Key Laboratory of Tissue Engineering, Shanghai, P.R. China

\section{References}

[1] Bloom, J. D, Antunes, M. B, \& Becker, D. G. Anatomy, physiology, and general concepts in nasal reconstruction. Facial Plast Surg Clin North Am (2011). , 19(1), 1-11.

[2] Immerman, S, White, W. M, \& Constantinides, M. Cartilage grafting in nasal reconstruction. Facial Plast Surg Clin North Am (2011). , 19(1), 175-82.

[3] Romo, T. rd, Pearson JM. Nasal implants. Facial Plast Surg Clin North Am (2008). vi., 16(1), 123-32.

[4] Graham, B. S, Thiringer, J. K, \& Barrett, T. L. Nasal tip ulceration from infection and extrusion of a nasal alloplastic implant. J Am Acad Dermatol (2001). Suppl):, 362-4.

[5] Langer, R, \& Vacanti, J. P. Tissue engineering. Science (1993). , 260(5110), 920-6.

[6] Zhang, L. It is time to reconstruct human auricle more precisely and microinvasively. Plast Reconstr Surg (2010). e-156e.

[7] Zhang, L, \& Spector, M. Tissue Engineering of Musculoskeletal Tissue. In: Pallua N, Suschek C, eds. Tissue Engineering: From Lab to Clinic:Springer,(2011).

[8] Cao, Y, Vacanti, J. P, Paige, K. T, Upton, J, \& Vacanti, C. A. Transplantation of chondrocytes utilizing a polymer-cell construct to produce tissue-engineered cartilage in the shape of a human ear. Plast Reconstr Surg (1997). discussion 303-4., 100(2), 297-302.

[9] Kamil, S. H, Kojima, K, Vacanti, M. P, Bonassar, L. J, Vacanti, C. A, \& Eavey, R. D. In vitro tissue engineering to generate a human-sized auricle and nasal tip. Laryngoscope (2003). , 113(1), 90-4.

[10] Cui, L, Wu, Y, Cen, L, Zhou, H, Yin, S, Liu, G, Liu, W, \& Cao, Y. Repair of articular cartilage defect in non-weight bearing areas using adipose derived stem cells loaded polyglycolic acid mesh. Biomaterials (2009). , 30(14), 2683-93.

[11] Frenkel, S. R. Di Cesare PE. Scaffolds for articular cartilage repair. Ann Biomed Eng (2004). , 32(1), 26-34. 
[12] Heath, C. A, \& Magari, S. R. Mini-review: Mechanical factors affecting cartilage regeneration in vitro. Biotechnol Bioeng (1996). , 50(4), 430-7.

[13] Aufderheide, A. C, \& Athanasiou, K. A. Comparison of scaffolds and culture conditions for tissue engineering of the knee meniscus. Tissue Eng (2005).

[14] Moran, J. M, Pazzano, D, \& Bonassar, L. J. Characterization of polylactic acid-polyglycolic acid composites for cartilage tissue engineering. Tissue Eng (2003). , 9(1), 63-70.

[15] Yan, D, Zhou, G, Zhou, X, Liu, W, Zhang, W. J, Luo, X, Zhang, L, Jiang, T, Cui, L, \& Cao, Y. The impact of low levels of collagen IX and pyridinoline on the mechanical properties of in vitro engineered cartilage. Biomaterials (2009). , 30(5), 814-21.

[16] Gunatillake, P. A, \& Adhikari, R. Biodegradable synthetic polymers for tissue engineering. Eur Cell Mater (2003). discussion 16., 5, 1-16.

[17] Kim, B. S, \& Mooney, D. J. Engineering smooth muscle tissue with a predefined structure. J Biomed Mater Res (1998). , 41(2), 322-32.

[18] Liu, Y, Zhang, L, Zhou, G, Li, Q, Liu, W, Yu, Z, Luo, X, Jiang, T, Zhang, W, \& Cao, Y. In vitro engineering of human ear-shaped cartilage assisted with CAD/CAM technology. Biomaterials (2010). , 31(8), 2176-83.

[19] Zhang, L, \& Spector, M. Comparison of three types of chondrocytes in collagen scaffolds for cartilage tissue engineering. Biomed Mater (2009).

[20] Bill, J. S, Reuther, J. F, Dittmann, W, Kubler, N, Meier, J. L, Pistner, H, \& Wittenberg, G. Stereolithography in oral and maxillofacial operation planning. Int J Oral Maxillofac Surg (1995). Pt 2):98-103.

[21] Ciocca, L, Mingucci, R, Gassino, G, \& Scotti, R. CAD/CAM ear model and virtual construction of the mold. J Prosthet Dent (2007). , 98(5), 339-43.

[22] Erickson, D. M, Chance, D, Schmitt, S, \& Mathis, J. An opinion survey of reported benefits from the use of stereolithographic models. J Oral Maxillofac Surg (1999). , 57(9), 1040-3.

[23] Subburaj, K, Nair, C, Rajesh, S, Meshram, S. M, \& Ravi, B. Rapid development of auricular prosthesis using CAD and rapid prototyping technologies. Int J Oral Maxillofac Surg (2007). , 36(10), 938-43.

[24] Al Mardini MErcoli C,Graser GN. A technique to produce a mirror-image wax pattern of an ear using rapid prototyping technology. J Prosthet Dent (2005). , 94(2), 195-8.

[25] Ciocca, L, \& Scotti, R. CAD-CAM generated ear cast by means of a laser scanner and rapid prototyping machine. J Prosthet Dent (2004). , 92(6), 591-5. 
[26] Karayazgan-saracoglu, B, Gunay, Y, \& Atay, A. Fabrication of an auricular prosthesis using computed tomography and rapid prototyping technique. J Craniofac Surg (2009). , 20(4), 1169-72.

[27] Yang, S, Leong, K. F, Du, Z, \& Chua, C. K. The design of scaffolds for use in tissue engineering. Part I. Traditional factors. Tissue Eng (2001). , 7(6), 679-89. 
